\title{
Long-term outcomes of infliximab in a real-world multicenter cohort of patients with acute severe ulcerative colitis
}

\author{
Shin Ju Oh${ }^{1}$, Ga Young Shin ${ }^{1}$, Hosim Soh ${ }^{2}$, Jae Gon Lee ${ }^{3}$, Jong Pil Im² , Chang Soo Eun ${ }^{3}$, Kang-Moon Lee ${ }^{4}$, Dong Il Park ${ }^{5}$, \\ Dong Soo Han ${ }^{3}$, Hyo Jong Kim ${ }^{1}$, Chang Kyun Lee ${ }^{1}$ \\ ${ }^{1}$ Center for Crohn's and Colitis, Department of Gastroenterology, Kyung Hee University Hospital, Kyung Hee University College of Medicine, \\ Seoul, ${ }^{2}$ Department of Internal Medicine and Liver Research Institute, Seoul National University Hospital, Seoul National University College \\ of Medicine, Seoul, ${ }^{3}$ Department of Gastroenterology, Hanyang University Guri Hospital, Hanyang University College of Medicine, Guri; \\ ${ }^{4}$ Department of Internal Medicine, St. Vincent's Hospital, College of Medicine, The Catholic University of Korea, Suwon; ${ }^{5}$ Department of Internal \\ Medicine, Kangbuk Samsung Hospital, Sungkyunkwan University School of Medicine, Seoul, Korea
}

Background/Aims: Infliximab (IFX) has proven effective as rescue therapy in steroid-refractory acute severe ulcerative colitis (ASUC), however, the long-term real-world data are scarce. Our study aimed to assess the long-term treatment outcomes of IFX in a real-life cohort. Methods: We established a multicenter retrospective cohort of hospitalized patients with ASUC, who met Truelove and Witt's criteria and received intravenous corticosteroid (IVCS) or IFX during index hospitalization between 2006 and 2016 in 5 university hospitals in Korea. The cohort was systematically followed up until colectomy, death or last follow-up visit. Results: A total of 296 patients were followed up for a mean of $68.9 \pm 44.0$ months. During index hospitalization, 49 patients were treated with IFX; as rescue therapy for IVCS failure in 37 and as first-line medical therapy for ASUC in 12. All patients treated with IFX avoided colectomy during index hospitalization. The cumulative rates of rehospitalization and colectomy were $20.4 \%$ and $6.1 \%$ at 3 months and $39.6 \%$ and $18.8 \%$ at the end of follow-up, respectively. Patients treated with IFX presented with significantly shorter colectomy-free survival than IVCS responders $(P=0.04$, log-rank test). Both cytomegalovirus colitis and Clostridioides difficile infection (CDI) were the significant predictors of colectomy in the overall study cohort (hazard ratios of 6.57 and 4.61, respectively). There were no fatalities. Conclusions: Our real-world cohort study demonstrated that IFX is an effective therapeutic option in Korean patients with ASUC, irrespective of IFX indication. Aggressive vigilance for cytomegalovirus colitis and CDI is warranted for hospitalized patients with ASUC. (Intest Res 2021;19:323-331)

Key Words: Colitis, ulcerative; Infliximab; Hospitalization; Colectomy

\section{INTRODUCTION}

Approximately $15 \%-25 \%$ of patients with ulcerative colitis (UC) may experience acute severe exacerbations, requiring immediate hospitalization and often surgical intervention

Received April 28, 2020. Revised June 2, 2020. Accepted June 3, 2020 Correspondence to Chang Kyun Lee, Center for Crohn's and Colitis, Department of Gastroenterology, Kyung Hee University College of Medicine, 23 Kyungheedae-ro, Dongdaemun-gu, Seoul 02447, Korea. Tel: +82-2-9588199, Fax: +82-2-958-8268, E-mail: changkyun.lee@khu.ac.kr during the course of the disease..$^{1-3}$ Treatment with intravenous corticosteroid (IVCS) during hospitalization is a mainstay in the treatment of acute severe UC (ASUC); however, $30 \%-40 \%$ of these patients do not respond to IVCS and, thus, require surgical resection or medical rescue therapy, such as cyclosporine and infliximab (IFX). ${ }^{4}$ A pivotal randomized controlled trial (RCT) comparing IFX with placebo demonstrated that a single infusion of IFX at $5 \mathrm{mg} / \mathrm{kg}$ was effective as rescue therapy in patients with moderately or severely active UC not responding to conventional treatment. ${ }^{5} \mathrm{~A}$ follow-up 
study to this pivotal RCT also demonstrated sustained benefits of IFX rescue therapy at 3 years. ${ }^{6}$

Despite the demonstrated efficacy of IFX rescue therapy in steroid-refractory ASUC, there exist only few long-term studies addressing the outcomes of IFX. In a Swedish study involving 211 hospitalized patients with steroid-refractory ASUC, the probability of colectomy-free survival was 0.64 (95\% confidence interval [CI], 0.57-0.70) at 12 months, 0.59 (95\% CI, 0.52$0.66)$ at 3 years and 0.53 (95\% CI, $0.44-0.61)$ at 5 years. ${ }^{7}$ In a recent cohort study including patients enrolled in a RCT comparing cyclosporine with IFX, colectomy-free survival rates were $69.1 \%$ (95\% CI, 56.9\%-81.3\%) at 1 year and $65.1 \%$ (95\% CI, $52.4 \%-77.8 \%)$ at 5 years in patients who received IFX. ${ }^{8}$

The effectiveness of IFX as rescue therapy in non-Caucasian populations with steroid-refractory ASUC is questionable. Epidemiologic studies have reported that Asian patients with UC seem to have more favorable disease courses than Caucasian patients. ${ }^{9,10}$ A recent meta-analysis of population-based cohort studies also reported that there is significant variation in outcomes of inflammatory bowel disease across ethnic groups, pointing to the hidden biology behind the variation. ${ }^{11}$ However, there is a paucity of real-world data addressing this issue, particularly in populations with continuously rising incidences of UC. ${ }^{12}$ We found several studies reporting on the efficacy and safety of IFX treatment in East Asian populations, including in Korea and Japan; however, specific data in subsets of hospitalized patients with ASUC were severely limited. ${ }^{13-15}$

In this context, we established a unique multicenter cohort of hospitalized patients with ASUC, who satisfied the criteria given by Truelove and Witts ${ }^{3}$ and were treated with IVCS or IFX during index hospitalization. The specific aims of our study were (1) to investigate the therapeutic outcomes of IFX treatment in a long-term setting and (2) to identify predictive factors for colectomy in our study cohort, as well as in a subset of patients who received IFX.

\section{METHODS}

\section{Study Population and Data Collection}

Eligible patients were those with UC who were admitted to hospital for the management of a colitis flare-up at 1 of 5 highvolume tertiary care inflammatory bowel disease (IBD) centers affiliated with university hospitals in Korea. We searched the electronic databases of participating hospitals for cases from January 2006 to September 2016 which met this condition. Diagnosis of UC was based on the clinical, endoscopic, and histologic criteria recommended by current guidelines. ${ }^{16,17}$ Patients with UC who met the Truelove and Witt's criteria ${ }^{3}$ of ASUC and received IVCS (hydrocortisone at $300-400 \mathrm{mg} /$ day or methylprednisolone at $60-80 \mathrm{mg}$ /day) or IFX induction as rescue therapy ( $5 \mathrm{mg} / \mathrm{kg}$ of body weight) during index hospitalization were included in the study (IFX-rescue). Medical rescue therapy with IFX was initiated when eligible patients showed inadequate clinical or biochemical responses after 3-7 days of IVCS administration. We found 12 patients with ASUC who were subjected to IFX as first-line therapy for ASUC, without first-line IVCS therapy because of systemic side effects to corticosteroids or steroid-refractoriness to oral corticosteroids at baseline. After detailed discussions about therapeutic options, those patients decided to be treated with IFX as first-line medical therapy for ASUC and were included in the study (IFX-initial). The minimum requirement for follow-up was at least 3 months after discharge of the index hospitalization.

The study cohort was followed up until colectomy, death, or the last follow-up visit after index hospitalization. Comprehensive biological and clinical data of the study cohort were collected. The presence of concomitant infection with cytomegalovirus (CMV) or Clostridioides difficile during index hospitalization was carefully investigated. Concomitant CMV infection was defined when either CMV inclusion bodies were detected by H\&E stain and/or immunohistochemistry or CMV DNA was detected by polymerase chain reaction (PCR) in colonic tissue specimens obtained by flexible sigmoidoscopy or colonoscopy. ${ }^{18} \mathrm{C}$. difficile infection (CDI) was defined as the presence of toxigenic $C$. difficile via stool toxin assay, PCR or toxigenic culture in stool specimens, in accordance with international guidelines. ${ }^{19}$ Disease activity was assessed according to the Mayo score ${ }^{20}$ and disease extent was categorized according to the Montreal classification. ${ }^{17}$

\section{Outcome Measurements}

Primary outcome variables were the cumulative rates of rehospitalization and colectomy at 3 months from index hospitalization and at the end of follow-up. Rehospitalization was defined as any hospital admission related to UC exacerbation or complications for more than 2 days after discharge from the index hospitalization. Additionally, clinical remission was assessed at both 3 months and 12 months. Clinical remission was defined as a Mayo score of 2 points or lower, with no individual subscore exceeding 1 point. Follow-up sigmoidoscopy or colonoscopy were recommended to the study patients at 
both 3 and 12 months after discharge from the index admission. Clinical outcomes of patients treated with IFX (IFX group) were compared with those of IVCS responders as the reference group. Subgroup comparisons were performed according to IFX indication (IFX-rescue vs. IFX-initial).

\section{Statistical Analysis}

All statistical analysis was performed using SAS 9.4 (SAS Institute Inc., Cary, NC, USA). We used chi-square or Fisher exact tests for categorical data and independent $t$-tests or Wilcoxon rank-sum tests for continuous data between the study groups. Data were described as means (ranges) for continuous data and numbers (percentages) for discrete data.

To identify clinical predictors of colectomy, we performed multivariable Cox's proportional hazards regression analysis with Firth's penalized-likelihood correction method, which included all the significant variables identified from univariate analysis. Results of the multivariable analysis were described as hazard ratios (HRs) and 95\% CIs. Colectomy-free survival defined as absence of colectomy was plotted using the Ka-
plan-Meier survival curve. Differences in survival between study subgroups were compared by the log-rank test. All $P$ values are two-tailed, and a value of $P<0.05$ was considered indicative of significance.

\section{Ethical Considerations}

This study was approved by the Institutional Review Board of the coordinating center (Kyung Hee University Hospital, Seoul, South Korea) and other participating hospitals. The informed consent was waived. The study protocol was registered at the Clinical Research Information Service (KCT0002799).

\section{RESULTS}

\section{Baseline Characteristics of the Study Cohort}

We identified 338 eligible patients with a colitis flare who were admitted to participating hospitals during the study period. After exclusion of 42 patients who did not meet the inclusion criteria ( 1 unclassified IBD, 5 follow-up less than 3 months, 36 not treated with IVCS or IFX), a total of 296 hospitalized pa-

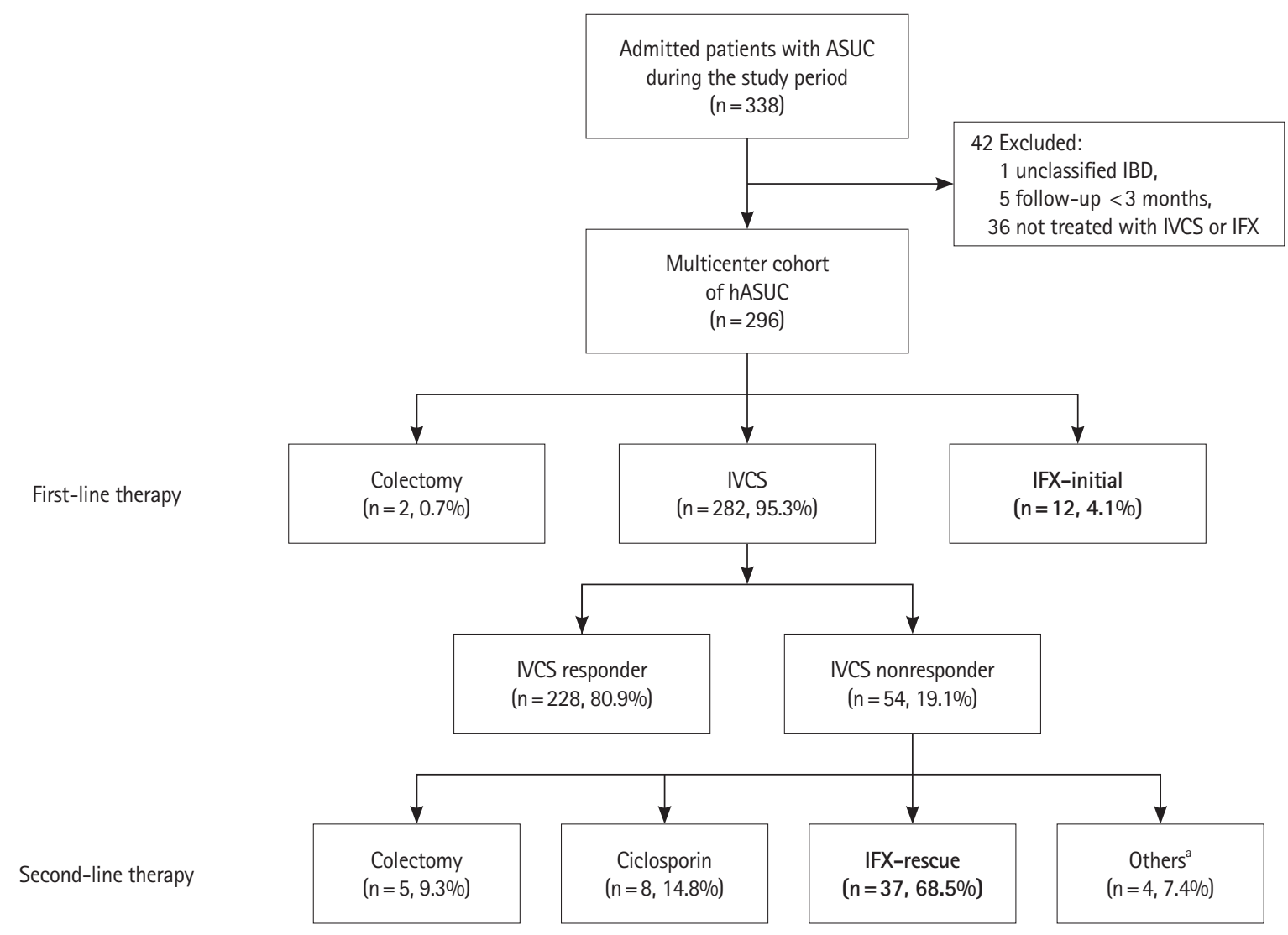

Fig. 1. Flowchart of the study. ${ }^{2}$ Others ( 3 adalimumab, 1 methorexate). ASUC, acute severe ulcerative colitis; IBD, inflammatory bowel disease; IFX, infliximab; IVCS, intravenous corticosteroid; hASUC, hospitalized patients with ASUC. 
tients with ASUC were finally included in our study (Fig. 1).

The mean follow-up period of the study cohort was $68.9 \pm 44.0$ months. Of the 296 patients in the study cohort, 282 (95.3\%) received IVCS and $12(4.1 \%)$ received IFX as first-line medical therapy for ASUC. The remaining 2 patients underwent emergency colectomies shortly after admission due to massive bleeding and colonic perforation, respectively. Approximately $80 \%$ of patients (228/282) showed clinical response to IVCS and avoided rescue therapy during index hospitalization. We termed these as "IVCS responders" and formed the reference group. Of 54 nonresponders to IVCS, 5 patients underwent colectomy

Table 1. Baseline Characteristics of the Study Population

\begin{tabular}{|c|c|c|c|}
\hline Demographic data & $\begin{array}{l}\text { IFX group } \\
(n=49)\end{array}$ & $\begin{array}{c}\text { IVCS } \\
\text { responder } \\
(n=228)\end{array}$ & $P$-value \\
\hline Age (yr) & $40.8 \pm 17.2$ & $44.5 \pm 16.7$ & 0.162 \\
\hline Male sex & $28(57.1)$ & $121(53.1)$ & $0.604^{a}$ \\
\hline Disease duration (mo) & $48.7 \pm 51.3$ & $48.7 \pm 99.9$ & 0.993 \\
\hline Smoking habits & & & $0.881^{\mathrm{a}}$ \\
\hline Never smoker & 40 (81.6) & $184(80.7)$ & \\
\hline Ex- or current smoker & $9(18.3)$ & 44 (19.3) & \\
\hline Family history of IBD & $1(2.0)$ & $5(2.2)$ & 1.000 \\
\hline Disease extent & & & $0.246^{a}$ \\
\hline E1 & $2(4.4)$ & $13(5.7)$ & \\
\hline E2 & $27(58.7)$ & $103(45.2)$ & \\
\hline E3 & $17(37.0)$ & $112(49.1)$ & \\
\hline Total Mayo score & $10.5 \pm 1.1$ & $10.7 \pm 1.3$ & 0.323 \\
\hline \multicolumn{4}{|l|}{ Laboratory findings } \\
\hline $\mathrm{CRP}(\mathrm{mg} / \mathrm{dL})$ & $4.3 \pm 5.0$ & $4.9 \pm 5.0$ & 0.495 \\
\hline Albumin (g/dL) & $3.6 \pm 0.7$ & $3.4 \pm 0.6$ & 0.099 \\
\hline $\mathrm{Hb}(\mathrm{g} / \mathrm{dL})$ & $11.7 \pm 1.9$ & $11.5 \pm 2.3$ & 0.501 \\
\hline $\operatorname{WBC}\left(\times 10^{3} / \mu \mathrm{L}\right)$ & $10.1 \pm 5.7$ & $9.8 \pm 3.8$ & 0.700 \\
\hline \multicolumn{4}{|l|}{ Concomitant treatments } \\
\hline Oral steroids & $31(63.3)$ & $79(34.7)$ & $<0.001^{a}$ \\
\hline Thiopurines & $21(42.9)$ & $28(12.3)$ & $<0.001$ \\
\hline Anti-TNF agents & 4 (8.2) & $8(3.5)$ & 0.235 \\
\hline Hospital stay (day) & $19.8 \pm 14.8$ & $14.1 \pm 10.1$ & 0.013 \\
\hline CMV colitis & $10(20.4)$ & 19 (8.3) & $0.012^{\mathrm{a}}$ \\
\hline C. difficile infection & $2(4.1)$ & $6(2.6)$ & 0.635 \\
\hline
\end{tabular}

Values are presented as mean \pm standard deviation or number (\%).

${ }^{\mathrm{a}}$ Fisher exact test.

IFX, infliximab; IVCS, intravenous corticosteroid; IBD, inflammatory bowel disease; CRP, C-reactive protein; Hb, hemoglobin; WBC, white blood cell; TNF,

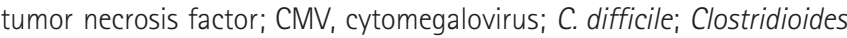
difficile. and 49 patients were treated with medical rescue therapy (37 with IFX, 8 with cyclosporin and 4 with other treatments, such as methotrexate and adalimumab). In total, 49 patients received IFX induction therapy as rescue or first-line treatment for ASUC during index hospitalization (the IFX group).

The baseline demographic and clinical characteristics of the reference and IFX groups are summarized in Table 1. In the IFX group, the mean age of patients was 40.8 years, $57 \%$ were male and the mean disease duration was 48.7 months. At index admission, the use of oral corticosteroids or thiopurines was significantly higher in the IFX group than in the reference group (63.3\% vs. $34.7 \%, 42.9 \%$ vs. $12.3 \%$, respectively, all $P<0.05)$. The mean hospital stay of the IFX group was 5.7 days longer than that of the reference group (19.8 days vs. 14.1 days, $P=$ 0.01). Compared to the reference group, the rate of simultaneous infection with CMV was significantly higher in the IFX group ( $20.4 \%$ vs. $8.3 \%, P=0.01$ ), but not the rate of CDI ( $4.1 \%$ vs. $2.6 \%, P=$ not significant). There were no significant side effects associated with IVCS or IFX during the index hospital stay in both groups.

\section{Short-term Outcomes}

All patients treated with IFX avoided colectomy during index hospitalization. At 3 months of follow-up, the rate of clinical remission was $26.1 \%$. The cumulative rates of rehospitalization and colectomy at 3 months were $20.4 \%$ and $6.1 \%$, respectively, in the IFX group. The cumulative rates of rehospitalization and colectomy at 3 months were $13.7 \%$ and 1.8\%, respectively, in the reference group.

\section{Long-term Outcomes}

At 12 months of follow-up, the rate of clinical remission was $45.5 \%$ in the IFX group. After a mean follow-up of $68.9 \pm 44.0$ months, the cumulative rates of rehospitalization and colectomy were $39.6 \%$ and $18.8 \%$, respectively, in the IFX group. The colectomy rate in the IFX group was significantly higher than in the reference group (18.8\% vs. $7.5 \%, P=0.026$ ) (Fig. 2). In Kaplan-Meier analysis, the IFX group had significantly shorter colectomy-free survival than the reference group $(P=0.04$ by log-rank test) (Fig. 3). No fatalities were observed in the overall study cohort.

\section{Subgroup Analysis of Clinical Outcomes}

There were no significant differences in either short- or longterm outcomes with respect to IFX treatment (IFX-rescue vs. IFX as first-line treatment subgroups) (Table 2). The mean 


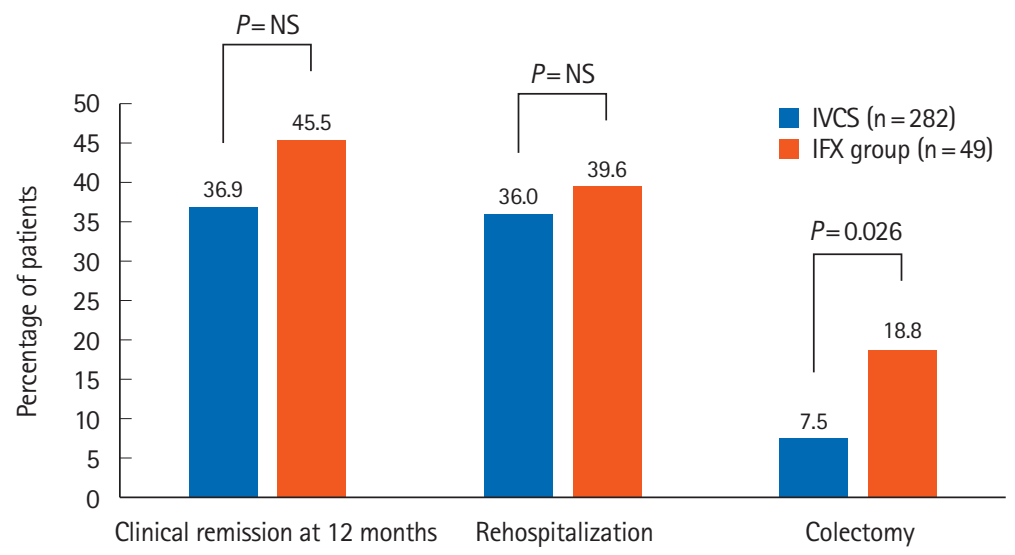

Fig. 2. Comparison of long-term outcomes between the study populations. IVCS, intravenous corticosteroid; IFX, infliximab.

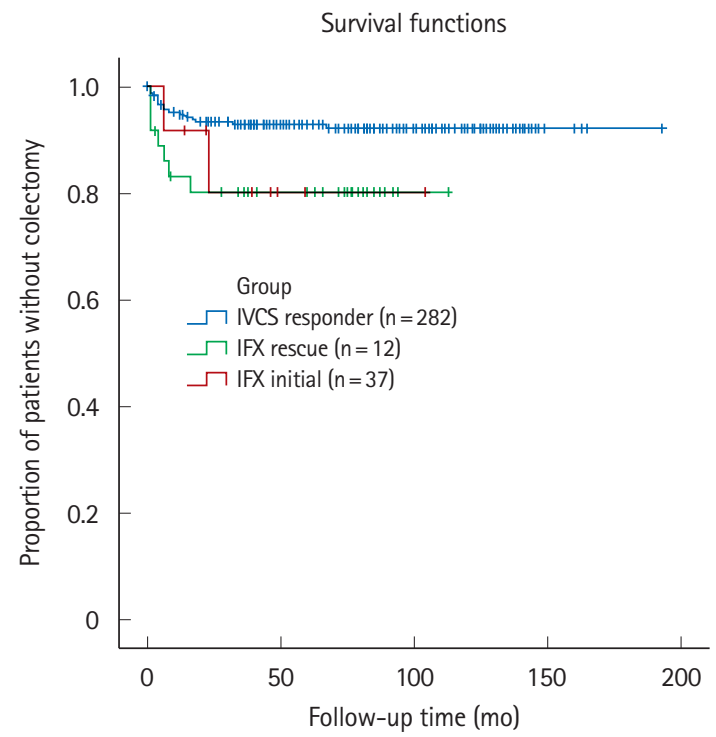

Fig. 3. Kaplan-Meier estimation of cumulative colectomy-free survival for the study populations (log-rank test, $P=0.036$ ). IVCS, intravenous corticosteroid; IFX, infliximab; IFX rescue, IFX as rescue therapy after failure of IVCS; IFX initial, IFX as first-line therapy for acute severe ulcerative colitis.

hospital stay during index hospitalization was significantly shorter in patients treated with IFX as first-line therapy than in those receiving IFX rescue therapy (11.2 days vs. 22.6 days, $P=$ $0.003)$.

In 12 patients treated with IFX as first-line therapy, 10 avoided colectomies during the follow-up period; 7 maintained IFX and 3 switched from IFX to adalimumab due to loss of response to IFX. Remaining 2 patients underwent colectomies during maintenance treatment with IFX, at 5 months and 24 months after discharge from the index hospitalization, respectively. In 37 patients with IFX as rescue therapy, 7 underwent colectomies ( 3 during induction period and 4 during mainte-
Table 2. Treatment Outcomes of IFX in Hospitalized Patients with Acute Severe Ulcerative Colitis, According to IFX Indication

\begin{tabular}{lccc}
\hline Variable & $\begin{array}{c}\text { IFX-rescue }^{\mathrm{a}} \\
(\mathrm{n}=37)\end{array}$ & $\begin{array}{c}\text { IFX-initial }^{\mathrm{b}} \\
(\mathrm{n}=12)\end{array}$ & $P$-value \\
\hline Short-term $^{\mathrm{c}}$ & & & \\
Clinical remission & $3(23.1)$ & $3(30.0)$ & 1.000 \\
Rehospitalizatioin $^{\text {Colectomy }}$ & $7(18.9)$ & $3(25.0)$ & 0.690 \\
Long-term $^{\mathrm{d}}$ & $3(8.1)$ & 0 & 0.566 \\
Clinical remission $^{\mathrm{e}}$ & $7(46.7)$ & $3(42.9)$ & 1.000 \\
Rehospitalization & $15(41.7)$ & $4(33.3)$ & 0.740 \\
Colectomy & $7(18.9)$ & $2(16.7)$ & 1.000 \\
\hline
\end{tabular}

Values are presented as number (\%).

Infliximab (IFX) as rescue therapy after failure of intravenous corticosteroids. ${ }^{b}$ IFX as first-line therapy for acute severe ulcerative colitis.

'At 3 months of follow-up.

${ }^{\mathrm{d}}$ At the end of follow-up.

${ }^{\mathrm{e}}$ At 12 months of follow-up.

nance with IFX). Remaining 30 patients avoided colectomies until the end of follow-up; 25 maintained IFX, 4 switched from IFX to adalimumab and 1 switched to another biologic drug during the follow-up period. In summary, 32 patients had successfully maintained IFX until the end of follow-up (retention rate of $65.3 \%$ ).

\section{Predictors of Colectomy}

Table 3 summarizes the results of multivariable Cox regression analysis aimed at uncovering predictors of colectomy in the overall cohort of hospitalized patients with ASUC. IFX administered as rescue therapy was the significant predictor of colectomy (HR, 2.80; 95\% CI, 1.09-7.17). In addition, previous failure of other anti-tumor necrosis factor (TNF) drugs, includ- 
Table 3. Predictors of Colectomy in Hospitalized Patients with Acute Severe Ulcerative Colitis

\begin{tabular}{lccc}
\hline Variable & HR & $95 \% \mathrm{Cl}$ & $P$-value \\
\hline IFX-rescue $^{\mathrm{a}}$ & 2.80 & $1.09-7.17$ & 0.032 \\
IFX-initial $^{\mathrm{b}}$ & 1.81 & $0.06-54.59$ & 0.732 \\
Failure of anti-TNFs $^{\mathrm{C}}$ & 4.90 & $1.62-14.84$ & 0.005 \\
CMV colitis $^{\text {C. difficile infection }}$ & 6.57 & $2.26-19.08$ & $<0.001$ \\
Duration of IVCS & 4.61 & $1.50-14.23$ & 0.008 \\
\hline
\end{tabular}

aIFX as rescue therapy after failure of intravenous corticosteroids.

IFX as first-line therapy for acute severe ulcerative colitis.

'Adalimumab or golimumab.

$\mathrm{HR}$, hazard ratio; $\mathrm{Cl}$, confidence interval; IFX, infliximab; TNF, tumor necrosis factor; CMV, cytomegalovirus; C. difficile, Clostridioides difficile; IVCS, intravenous corticosteroid.

ing adalimumab and golimumab, duration of IVCS administration during index hospitalization, CMV colitis, and CDI were associated with a higher risk of colectomy (HR, 4.90; 95\% CI, 1.62-14.84; HR, 1.07; 95\% CI, 1.00-1.15; HR, 6.57; 95\% CI, 2.26-19.08; HR, 4.61; 95\% CI, 1.50-14.23, respectively).

\section{DISCUSSION}

The present study focused on the treatment outcomes of IFX in Asian patients with ASUC in a long-term set-up. Our study cohort comprised patients from 5 university hospitals with a large volume of patients with IBD in Korea, where the incidence of UC has been continuously rising in recent years. ${ }^{21}$ The study cohort was systematically followed up for a long period-for a mean follow-up of $68.9 \pm 44.0$ months.

Here, we observed that patients treated with IFX showed more favorable short-term as well as long-term outcomes than those reported in previous studies. Irrespective of IFX indication, all patients that received IFX induction therapy avoided colectomy during the index hospitalization. Specifically, the clinical response rates to IFX rescue therapy in our study were $79.2 \%$ and $73.7 \%$ at 3 months and 1 year, respectively (data not provided). The results are comparable to or slightly better from those reported in a recent systematic review, in which clinical response rates were reported as $46 \%-83 \%$ at 3 months and 50\%-65\% 1 year after IFX rescue therapy. ${ }^{2}$ The colectomy rates after IFX rescue therapy in our study were $8.1 \%$ and $18.9 \%$ at 3 months and at the end of follow-up, respectively, which are also much lower than those reported in recent studies but similar to those reported in a study conducted in the same country. ${ }^{2,8,15,22}$ A multicenter retrospective study conducted in the United States reported that the rates of in-hospital colectomy were $8 \%, 27 \%$, and $32 \%$ at 3,12 , and 24 months, respectively, after IFX rescue therapy for ASUC. ${ }^{22}$ In a European multicenter cohort study involving 55 patients with ASUC, the colectomy-free survival rate was $61.5 \%$ (95\% CI, $52.4 \%-77.8 \%$ ) at 5 years after IFX rescue therapy. ${ }^{8}$ In summary, our data indicate that IFX is an effective treatment option for avoiding colectomy in both short-term and long-term settings in non-Caucasian populations with ASUC. The current results also support a recent observation that the Korean population with UC may have a more favorable natural history than Caucasians. ${ }^{23}$

In this study, we reported on the clinical course of a subgroup of patients who were treated with IFX as first-line medical therapy for ASUC (IFX-initial) due to systemic side effects or steroid-refractoriness to oral corticosteroids at baseline. As described previously, all these patients avoided colectomy during index hospitalization. None of the patients were subjected to colectomy within 3 months of follow-up (Table 2). Although our data need further verification in a larger number of patients, we believe that the current results provide a practical guide for clinicians to consider IFX as first-line medical therapy in selected patients with ASUC if they have pre-existing steroid-refractoriness or intolerance at admission. However, clinicians should keep in mind that the long-term clinical course in this subset of patients was not similar with that of the IVCS responders but was similar to that of patients receiving IFX rescue therapy (Fig. 3).

Notwithstanding the favorable results reported above, we demonstrated that the colectomy-free survival rate in IFXtreated patients was significantly shorter than that in IVCS responders, pointing to the need for further efforts to improve treatment outcomes of IFX. Patient stratification must be a first target for future investigations. In our study, significantly more patients treated with IFX were using oral corticosteroids or thiopurines at baseline, as compared to IVCS responders (63.3\% vs. $34.7 \%, 42.9 \%$ vs. $12.3 \%$, respectively, all $P<0.05$ ). These results suggest those patients might benefit from early or aggressive introduction of IFX. ${ }^{24,25}$

Our multivariable Cox regression analysis has identified multiple independent predictors of colectomy in the overall study cohort (Table 3 ). In addition to IFX given as rescue therapy (HR, 2.80; 95\% CI, 1.09-7.17), we found that, at baseline, previous failure of anti-TNF drugs (adalimumab or golimumab), simultaneous CMV colitis, and concurrent CDI were the major predictors of colectomy (HR, 4.90; 95\% CI, 1.62-14.84; HR, 6.57; 95\% CI, 2.26-19.08; HR, 4.61; 95\% CI, 1.50-14.23, re- 
spectively). Previous failure of first anti-TNF drugs in IBD is a well-known independent risk factor for failure of other antiTNF drugs. ${ }^{26}$ Therefore, it appears reasonable to conclude that previous failure of anti-TNF drugs might be associated with suboptimal responses to IFX therapy and subsequent colectomy in our study cohort. More importantly, our results add further weight to the prognostic importance of CMV colitis in hospitalized patients with ASUC. In the present study, we demonstrated that CMV colitis was the most potent predictor of colectomy in the overall study cohort (HR, 6.57; 95\% CI, 2.26-19.08). These results are in line with our previous report, in that true CMV colitis verified in colonic tissue was an independent predictor of poor disease outcomes (hospitalization and colectomy) in patients with moderate to severe UC flareups. ${ }^{27}$ The present study strongly suggests the need for immediate treatment with antivirals in CMV colitis superimposed on ASUC. Antiviral therapy for CMV colitis complicating UC, however, remains a controversial issue ${ }^{18}$ Further prospective studies are required to validate our findings. Lastly, the adverse outcomes of CDI complicating IBD have been well described, but data regarding the impact of CDI on risk of colectomy are still limited. A recent meta-analysis of 12 observational studies reported that CDI appears to increase long-term colectomy risk in patients with UC (odds ratio, 2.96; 95\% CI, 1.19-7.34) ${ }^{28}$ Here, we demonstrated that CDI was one of the significant predictors of colectomy in the overall study cohort with ASUC (HR, 4.61; 95\% CI, 1.50-14.23). Our findings bolster the relevant clinical implication that CDI should be more aggressively screened for and managed in hospitalized patients with ASUC, particularly when considering IFX therapy.

Our study has several limitations derived from the retrospective nature of the study design. First, clinical practice might have varied across the participating hospitals during the long study period. Second, our study did not provide data regarding endoscopic remission with IFX treatment. It is reported that mucosal healing with IFX is associated with long-term prognosis of $\mathrm{UC}^{29}$ and therefore mucosal healing has been established as one of important therapeutic goals in UC. Unfortunately, our study was not specifically designed to answer the question of whether achieving mucosal healing leads to better outcomes in hospitalized patients with ASUC. Further, our sample size of patients with ASUC, particularly those treated with IFX was relatively small compared to those of Western studies. Several studies have described the long-term efficacy of IFX in East Asian population with UC; however, these studies were heterogeneous in their designs and patient popula- tions. ${ }^{13-15,30,31}$ Large prospective studies with standardized protocols in different Asian populations with ASUC are therefore needed to overcome these limitations. Further clinical trials with different biologics or small molecules are also needed in this setting. ${ }^{32,33}$

In conclusion, we have reported the favorable short-term and long-term outcomes of IFX treatment in Korean patients with ASUC, pointing to IFX as an effective therapeutic option in such a critical situation. We believe that IFX can be considered as a first-line option for the treatment of ASUC, in highly selected patients, who cannot receive initial therapy with IVCS. Aggressive vigilance for both concurrent CMV colitis and CDI is warranted for hospitalized patients with ASUC, particularly when considering IFX therapy.

\section{ADDITIONAL INFORMATION}

\section{Funding Source}

This research was supported by Medical Research Center Program through the National Research Foundation of Korea (NRF) funded by the Ministry of Science and ICT (NRF-2017R1A5A2014768).

\section{Conflict of Interest}

Park DI and Han DS are editorial board members of the journal but were not involved in the peer reviewer selection, evaluation, or decision process of this article. No other potential conflicts of interest relevant to this article were reported.

\section{Author Contribution}

Conceptualization: Oh SJ, Lee CK. Data curation: all authors. Formal analysis: Oh SJ, Lee CK. Funding acquisition: Kim HJ. Investigation: Shin GY, Soh H, Lee JG, Im JP, Eun CS, Lee KM, Park DI, Han DS, Kim HJ, Lee CK. Methodology: Lee CK. Writing - original draft: Oh SJ, Lee CK. Writing - review \& editing: all authors. Approval of final manuscript: all authors.

\section{ORCID}

Oh SJ

Shin GY

Soh $\mathrm{H}$

Lee JG

Im JP

Eun CS

Lee KM

Park DI https://orcid.org/0000-0003-0470-1026 https://orcid.org/0000-0001-7869-9737 https://orcid.org/0000-0001-5107-6521 https://orcid.org/0000-0003-2930-8159 https://orcid.org/0000-0003-1584-0160 https://orcid.org/0000-0001-6533-9644 https://orcid.org/0000-0003-2850-4553 https://orcid.org/0000-0003-2307-8575 
Han DS

$\mathrm{Kim} \mathrm{HJ}$

Lee CK

https://orcid.org/0000-0001-7103-3318

https://orcid.org/0000-0002-9675-4557

https://orcid.org/0000-0002-4279-3825

\section{REFERENCES}

1. Dinesen LC, Walsh AJ, Protic MN, et al. The pattern and outcome of acute severe colitis. J Crohns Colitis 2010;4:431-437.

2. Seah D, De Cruz P. Review article: the practical management of acute severe ulcerative colitis. Aliment Pharmacol Ther 2016;43:482-513.

3. Truelove SC, Witts LJ. Cortisone in ulcerative colitis: final report on a therapeutic trial. Br Med J 1955;2:1041-1048.

4. Turner D, Walsh CM, Steinhart AH, Griffiths AM. Response to corticosteroids in severe ulcerative colitis: a systematic review of the literature and a meta-regression. Clin Gastroenterol Hepatol 2007;5:103-110.

5. Järnerot $\mathrm{G}$, Hertervig E, Friis-Liby I, et al. Infliximab as rescue therapy in severe to moderately severe ulcerative colitis: a randomized, placebo-controlled study. Gastroenterology 2005;128:1805-1811.

6. Gustavsson A, Järnerot G, Hertervig E, et al. Clinical trial: colectomy after rescue therapy in ulcerative colitis: 3-year followup of the Swedish-Danish controlled infliximab study. Aliment Pharmacol Ther 2010;32:984-989.

7. Sjöberg M, Magnuson A, Björk J, et al. Infliximab as rescue therapy in hospitalised patients with steroid-refractory acute ulcerative colitis: a long-term follow-up of 211 Swedish patients. Aliment Pharmacol Ther 2013;38:377-387.

8. Laharie D, Bourreille A, Branche J, et al. Long-term outcome of patients with steroid-refractory acute severe UC treated with ciclosporin or infliximab. Gut 2018;67:237-243.

9. Park SH, Kim YM, Yang SK, et al. Clinical features and natural history of ulcerative colitis in Korea. Inflamm Bowel Dis 2007; 13:278-283.

10. Fumery M, Singh S, Dulai PS, Gower-Rousseau C, Peyrin-Biroulet L, Sandborn WJ. Natural history of adult ulcerative colitis in population-based cohorts: a systematic review. Clin Gastroenterol Hepatol 2018;16:343-356.

11. Shi HY, Levy AN, Trivedi HD, Chan FKL, Ng SC, Ananthakrishnan AN. Ethnicity influences phenotype and outcomes in inflammatory bowel disease: a systematic review and meta-analysis of population-based studies. Clin Gastroenterol Hepatol 2018;16:190-197.

12. Ng WK, Wong SH, Ng SC. Changing epidemiological trends of inflammatory bowel disease in Asia. Intest Res 2016;14:111-
119.

13. Seo H, Chang K, Lee SH, et al. Long-term outcomes of infliximab treatment and predictors of response in 195 patients with ulcerative colitis: a hospital-based cohort study from Korea. Scand J Gastroenterol 2017;52:857-863.

14. Minami N, Yoshino T, Matsuura M, et al. Tacrolimus or infliximab for severe ulcerative colitis: short-term and long-term data from a retrospective observational study. BMJ Open Gastroenterol 2015;2:e000021.

15. Lee KM, Jeen YT, Cho JY, et al. Efficacy, safety, and predictors of response to infliximab therapy for ulcerative colitis: a Korean multicenter retrospective study. J Gastroenterol Hepatol 2013;28:1829-1833.

16. Choi CH, Jung SA, Lee BI, et al. Diagnostic guideline of ulcerative colitis. Korean J Gastroenterol 2009;53:145-160.

17. Magro F, Gionchetti P, Eliakim R, et al. Third European evidence-based consensus on diagnosis and management of ulcerative colitis. Part 1: definitions, diagnosis, extra-intestinal manifestations, pregnancy, cancer surveillance, surgery, and ileo-anal pouch disorders. J Crohns Colitis 2017;11:649-670.

18. Beswick L, Ye B, van Langenberg DR. Toward an algorithm for the diagnosis and management of CMV in patients with colitis. Inflamm Bowel Dis 2016;22:2966-2976.

19. McDonald LC, Gerding DN, Johnson S, et al. Clinical practice guidelines for clostridium difficile infection in adults and children: 2017 update by the Infectious Diseases Society of America (IDSA) and Society for Healthcare Epidemiology of America (SHEA). Clin Infect Dis 2018;66:e1-e48.

20. Schroeder KW, Tremaine WJ, Ilstrup DM. Coated oral 5-aminosalicylic acid therapy for mildly to moderately active ulcerative colitis. A randomized study. N Engl J Med 1987;317:16251629.

21. Park SH, Kim YJ, Rhee KH, et al. A 30-year trend analysis in the epidemiology of inflammatory bowel disease in the Songpa-Kangdong District of Seoul, Korea in 1986-2015. J Crohns Colitis 2019;13:1410-1417.

22. Nalagatla N, Falloon K, Tran G, et al. Effect of accelerated infliximab induction on short- and long-term outcomes of acute severe ulcerative colitis: a retrospective multicenter study and meta-analysis. Clin Gastroenterol Hepatol 2019;17:502-509.

23. Lee HS, Yang SK, Soh JS, et al. Short- and long-term outcomes of acute severe ulcerative colitis in Korea: the 1999-2005 cohort. Inflamm Bowel Dis 2015;21:1825-1831.

24. Gibson DJ, Heetun ZS, Redmond CE, et al. An accelerated infliximab induction regimen reduces the need for early colectomy in patients with acute severe ulcerative colitis. Clin Gas- 
troenterol Hepatol 2015;13:330-335.

25. Seah D, Choy MC, Gorelik A, et al. Examining maintenance care following infliximab salvage therapy for acute severe ulcerative colitis. J Gastroenterol Hepatol 2018;33:226-231.

26. Gisbert JP, Chaparro M. Predictors of primary response to biologic treatment (anti-TNF, vedolizumab and ustekinumab) in patients with inflammatory bowel disease: from basic science to clinical practice. J Crohns Colitis 2020;14:694-709.

27. Oh SJ, Lee CK, Kim YW, et al. True cytomegalovirus colitis is a poor prognostic indicator in patients with ulcerative colitis flares: the 10-year experience of an academic referral inflammatory bowel disease center. Scand J Gastroenterol 2019; 54:976-983.

28. Law CC, Tariq R, Khanna S, Murthy S, McCurdy JD. Systematic review with meta-analysis: the impact of Clostridium difficile infection on the short- and long-term risks of colectomy in inflammatory bowel disease. Aliment Pharmacol Ther 2017;45: 1011-1020.

29. Colombel JF, Rutgeerts P, Reinisch W, et al. Early mucosal heal- ing with infliximab is associated with improved long-term clinical outcomes in ulcerative colitis. Gastroenterology 2011; 141:1194-1201.

30. Saigusa K, Matsuoka K, Sugimoto S, et al. Ulcerative colitis endoscopic index of severity is associated with long-term prognosis in ulcerative colitis patients treated with infliximab. Dig Endosc 2016;28:665-670.

31. Nasuno M, Miyakawa M, Tanaka H, Motoya S. Short- and long-term outcomes of infliximab treatment for steroid-refractory ulcerative colitis and related prognostic factors: a single-center retrospective study. Digestion 2017;95:67-71.

32. Berinstein JA, Steiner CA, Regal RE, et al. Efficacy of induction therapy with high-intensity tofacitinib in 4 patients with acute severe ulcerative colitis. Clin Gastroenterol Hepatol 2019;17: 988-990.

33. Kotwani P, Terdiman J, Lewin S. Tofacitinib for rescue therapy in acute severe ulcerative colitis: a real-world experience. J Crohns Colitis 2020;14:1026-1028. 